\title{
One-Year Evolution of Symptoms and Health Status of the COPD Multi-Dimensional Phenotypes: Results from the Follow-Up of the STORICO Observational Study
}

This article was published in the following Dove Press journal:

International Journal of Chronic Obstructive Pulmonary Disease

\author{
Raffaele Antonelli Incalzi ${ }^{1}$ \\ Francesco Blasi $\mathbb{D D}^{2,3}$ \\ Nicola Scichilone (iD ${ }^{4}$ \\ Alessandro Zullo ${ }^{5}$ \\ Lucia Simoni $^{5}$ \\ Giorgio Walter Canonica ${ }^{6}$ \\ On Behalf of STORICO \\ study group
}

'Internal Medicine and Geriatrics Department Biomedical Campus University of Rome, Rome, Italy; ${ }^{2}$ Internal Medicine Department, Respiratory Unit and Cystic Fibrosis Adult Center, Fondazione IRCCS Cà Granda Ospedale Maggiore Policlinico, Milan, Italy; ${ }^{3}$ Department of Pathophysiology and Transplantation, University of Milan, Milan, Italy; ${ }^{4}$ DIBIMIS, University of Palermo, Palermo, Italy; ${ }^{5}$ Medineos Observational Research, Modena, Italy; ${ }^{6}$ Personalized Medicine Asthma \& Allergy Clinic, Humanitas University, Humanitas \& Research Hospital-IRCCS, Rozzano (Milan), Italy
Correspondence: Raffaele Antonelli Incalzi Biomedical Campus, University of Rome, Via Alvaro Del Portillo, 2I, Rome, 00128, Italy Tel +3906 2254l I365

Fax $+39062254 \mid 456$

Email papermultiSTORICO@medineos.com
Aim: Describing the 1-year evolution of symptoms and health status in COPD patients enrolled in the STORICO study (observational study on characterization of 24-h symptoms in patients with COPD) classified in multidimensional phenotypes (m-phenotypes).

Methods: In our previous study, we performed an exploratory factor analysis to identify clinical and pathophysiological variables having the greatest classificatory properties, followed by a cluster analysis to group patients into m-phenotypes (mild COPD (MC), mild emphysematous (ME), severe bronchitic (SB), severe emphysematous (SE), and severe mixed COPD (SMC)). COPD symptoms were recorded at baseline, 6-, and 12-month followup and their evolution was described as frequency of patients with always present, always absent, arising', 'no more present symptoms. QoL and quality of sleep were evaluated using the SGRQ and CASIS questionnaires, respectively.

Results: We analyzed 379 subjects (144 MC, 71 ME, 96 SB, 14 SE, 54 SMC). M-phenotypes were stable over time in terms of presence of symptoms and health status with selected differences in evolution of symptoms in mild vs severe m-phenotypes. Indeed, $28.1 \% \mathrm{SB}, 50.0 \% \mathrm{SE}$ and $24.1 \% \mathrm{SMC}$ vs $0.7 \% \mathrm{MC}$ and $5.6 \% \mathrm{ME}$ with nighttime symptoms at baseline had no more symptoms at 6-month (p-value night-time symptom evolution $\mathrm{MC}$ vs $\mathrm{SB}, \mathrm{SE}, \mathrm{SMC}$ and $\mathrm{ME}$ vs $\mathrm{SB}, \mathrm{SE}, \mathrm{SMC}<0.0001$ ). All m-phenotypes improved in quality of sleep, more markedly the severe than the mild ones (p-values CASIS score change between baseline and 6- or 12-month in MC, ME vs SB, SE, SMC <0.0001). QoL did not change during observation, irrespectively of m-phenotype.

Conclusion: Over 1 year, severe m-phenotypes showed an improvement in night-time symptoms and quality of sleep, but not QoL. Being stable over time, m-phenotypes seem worthy of testing for classificatory and prognostic purposes.

Keywords: phenotype, evolution, quality of sleep, quality of life, real world, cohort study

\section{Introduction}

Chronic Obstructive Pulmonary Disease (COPD) is an umbrella definition encompassing a variety of clinical conditions with different pathology and pathophysiological bases. ${ }^{1}$ Thus, many attempts have been made and are ongoing to sort out phenotypes, or at least treatable traits, in order to tailor the therapy to the true needs of the patients. $^{2-6}$ 
Such an objective has been originally pursued by identifying clinical phenotypes (mainly bronchitic, emphysematous, asthma-like, frequent exacerbator). ${ }^{7,8}$ Increasing awareness of clinical variability within individual clinical phenotypes and of the role of comorbid diseases in conditioning the health status have prompted alternative, more complex classificatory strategies variably including biological markers. This marks the passage from the phenotype to the endotype. ${ }^{9}$ Complex analytical procedures, such as network analysis and machine learning, have been used to improve classification. ${ }^{10-13}$ However, these classifications, by focusing on systemic features of COPD and comorbid diseases, carry some risk of neglecting core respiratory symptoms. Indeed, dyspnea, cough and phlegm remain the triad characterizing the COPD patient, even if other symptoms, like fatigue and depressed mood, are highly prevalent and disabling. ${ }^{14}$

At variance with the numerous comprehensive "systemic" classifications of COPD recently proposed, the STORICO study (STudio Osservazionale sulla caratteRizzazione dei sIntomi delle 24 ore nei pazienti con broncopneumopatia cronica ostruttiva, observational study on characterization of 24-h symptoms in patients with COPD) attempts to overcome the recognized weakness of the traditional clinical classification by deepening the clinical assessment rather than making it an almost marginal component of a multidimensional assessment. The well-recognized classificatory properties of symptoms, if properly assessed, ie, if collected in their circadian sequence, constitute the foundations of the philosophy of the STORICO study. ${ }^{15}$ Furthermore, assessing the circadian rhythm of symptoms allows an evaluation of the classificatory properties of nocturnal symptoms. Commonly neglected in the clinical assessment of COPD patients, night-time symptoms are worthy of attention as they have many determinants, they significantly impact the health status and have potential prognostic implications. ${ }^{16}$ Indeed, the baseline assessment of a real-life COPD population enabled us to identify 5 cluster of patients (named multidimensional phenotypes, hereinafter named m-phenotypes) with distinctive clinical profiles built up by the analysis of the circadian rhythm of symptoms and the quality of life rated by the Saint George Respiratory Questionnaire (SGRQ). These clusters were characterized by quantitatively and qualitatively different burdens of disease, which might pave the way to tailored therapies as well as to goal-directed treatment.
The present analysis aimed to describe the 1-year clinical evolution (in terms of circadian rhythm of symptoms, occurrence of exacerbations, and lung function) and health status (in terms of quality of life, quality of sleep, and level of anxiety and depression) in COPD patients classified according to m-phenotypes.

\section{Methods}

\section{Study Design and Population}

STORICO (study registration number: NCT03105999) is an Italian, multicenter, observational study conducted in 40 pulmonology centers. Patients were consecutively recruited from February 2016 to April 2017 and followup visits were scheduled after 6 and 12 months from baseline. Last patient last visit occurred in June 2018. The study was approved by the Ethic Committee of the coordinating center (Fondazione Toscana G. Monasterio Pisa, Italy) and was conducted in accordance with the Declaration of Helsinki and the Good Clinical Practices guidelines for observational studies, in compliance with all the requirements of Italian regulations. Patients provided written, informed consent before study participation.

Subjects enrolled were aged $\geq 50$, current or exsmokers with smoking history $\geq 10$ pack-years, with a diagnosis of COPD, free from exacerbations (defined according to GOLD $2014^{17}$ guidelines) at enrolment visit and in the month before.

For the present analysis, we considered patients who completed baseline and follow-up visits within $6( \pm 2)$ and $12( \pm 3)$ months, respectively, and with available information on the frequency of COPD symptoms during each part of the 24-h day at baseline and both follow-up visits. The methodology of the STORICO study has been fully described elsewhere. ${ }^{15}$

Patients were classified at enrollment into five m-phenotypes, through a series of steps that have been extensively described in our previous paper. ${ }^{17}$

These steps can be briefly summarized here as follows: (i) An exploratory factor analysis (EFA) was performed to find independent latent constructs (factors), not directly measurable and influencing responses on observed variables. EFA is a variable reduction technique which does not impose any preconceived structure on the outcome and the observed variables included in the model are a linear combination of the underlying factors. The following items from the symptoms questionnaire were included in the factor analysis: presence/absence of shortness of breath 
or breathlessness, cough, bringing up phlegm or mucus, each symptom evaluated during the 24 hours (ie, in the early-morning, day-time and night-time). The value of the FEV1\% predicted, (symptoms, activity, impact) SGRQ scores, demographic variables (age and gender), and presence/absence of relevant comorbidities (cardiac ischemic disease, arterial hypertension, heart failure, atrial fibrillation, diabetes, osteoporosis, depression, kidney insufficiency) were also included in the model. Orthogonal VARIMAX rotation was applied; factors having an eigenvalue $>1.0$ and individual variables with higher-than-0.5 loadings on retained factors were retained. EFA identified 2 factors and, with them, the clinical and pathophysiological variables having the greatest classificatory properties (ie, the circadian rhythm of symptoms (dyspnea, bringing up phlegm or mucus and cough) and quality of life (as assessed by the SGRQ)). (ii) Then, the variables included in the two factors were evaluated and, whenever possible, combined into a new classificatory variable (a classificatory variable for each factor was defined). (iii) A cluster analysis with classificatory variables mentioned at step (ii) as input was performed, and patients were thus grouped into m-phenotypes. Average linkage was chosen as clustering method and average distance between clusters equal to 0.70 was taken as reference to cut the dendrogram.

These m-phenotypes were denominated according to the dominant clinical trait as "emphysematous" or "bronchitic" depending upon whether dyspnea or productive cough (bringing up phlegm or mucus) was the dominant symptom, and "mixed", in case both "emphysematous" and "bronchitic" traits were present.

The m-phenotypes were, therefore, the following: mild COPD (MC), characterized by absence of night-time symptoms without a prevalent bronchitic or emphysematous trait, mild emphysematous (ME), with prevalent dyspnea in the early-morning and day-time, but not by night, severe bronchitic (SB), with nocturnal and diurnal cough and phlegm, but no nocturnal dyspnea, severe emphysematous (SE), presenting nocturnal and diurnal dyspnea and severe mixed COPD (SMC), reporting the higher frequency of symptoms during $24 \mathrm{~h}$.

\section{Clinical Assessment}

At baseline, socio-demographic characteristics and clinical history were recorded.

At study visits, spirometry was performed according to the recommendations of the American Thoracic Society and of the European Respiratory Society: forced expiratory volume in the first second (FEV1) and Forced Vital Capacity (FVC) were collected, and, where available, Residual Volume (RV), Total Lung Capacity (TLC), and diffusion capacity for carbon monoxide (DLCO) were also recorded.

An exacerbation was defined as an acute event characterized by a worsening of the patient's respiratory symptoms beyond day-to-day variations which led to a change in medication during follow-up. At enrolment visit, anamnestic data about number of COPD exacerbations/year in the 5 years before enrolment were collected by the clinical investigator. The occurrence and severity of exacerbations during the study were assessed as well.

\section{Outcome Measures}

The frequency of COPD symptoms (breathlessness, coughing, bringing up phlegm or mucus, chest tightness, chest congestion, and wheezing) during each part of the day (early-morning, day-time, and night-time) was assessed at study visits by means of the Night-time, Morning, and Daytime Symptoms of COPD questionnaire. ${ }^{18}$ The level of perceived breathlessness and the extent to which it affected mobility were assessed using the modified Medical Research Council (mMRC) dyspnea scale ranging from 0 (breathless with strenuous exercise) to 4 (too breathless to leave the house/breathless when dressing or undressing). ${ }^{19}$ Presence of dyspnea was defined as a mMRC scale score $\geq 2$.

QoL was evaluated using the SGRQ, including the subject's perception of recent respiratory problems (Symptoms component), disturbances to daily physical activity (Activity component), and disturbances of psychosocial function (Impact component); total score was also calculated. Scores range between 0 (no impairment) and 100 (highest impairment). ${ }^{20,21}$

Anxiety and depression states were investigated through the Hospital Anxiety and Depression Scale (HADS), ${ }^{22}$ with a total score (emotional distress) ranging between 0 and 42; anxiety and depression subscale scores (ranging 0-21) were also computed, with higher scores indicating more distress.

The impact of respiratory symptoms on sleep was assessed with the COPD and Asthma Sleep Impact Scale (CASIS), ${ }^{23}$ a self-administered, 7-item scale evaluating sleep impairment associated with COPD and asthma. The total score ranges 0-100 (higher scores indicating greater sleep deterioration). Permissions have been obtained from authors for the use of the questionnaires/scales. 


\section{Data Quality Assurance}

The data entered into the electronic case report forms (eCRFs) by investigational staff were reviewed for completeness and accuracy and site personnel was instructed to enter data into the eCRFs and make any required corrections or additions during the phone training.

The scales and questionnaires were filled out by patients and the data were entered into the eCRFs by qualified personnel. The eCRF was provided with online edit checks. Two cleaning waves were performed by running post-entry checks by means of validation programs and data listings specific for the study. During this process, if clarifications were needed, queries were raised through the eCRF. Designated investigator site personnel was required to respond to the query and make the correction to the database.

\section{Statistical Analysis}

Our analysis was descriptive and no formal statistical hypotheses were set. The continuous, normally distributed variables were expressed as a mean \pm standard deviation (SD) or median and interquartile range (IQR) in case of not normally distributed parameters, while absolute and relative frequencies were provided for qualitative data. Missing values were not replaced and did not contribute to the analysis of the variable.

According to the Night-time, Morning, and Day-time Symptoms of COPD questionnaire, presence of COPD symptoms in the early-morning (or in the night-time or day-time) was defined as occurrence of at least one symptom (among breathlessness, coughing, bringing up phlegm or mucus, chest tightness, chest congestion, and wheezing) in the early-morning (or in the night-time or day-time) during the week before visit, irrespective of severity and frequency.

The evolution of (night-time, early-morning, and daytime) COPD symptoms during follow-up was described in terms of frequency of patients with symptoms: (i) "always present", ie, present at two subsequent visits (baseline and 6-month follow-up or 6- and 12-month follow-up), (ii) "always absent", ie, absent at two subsequent visits (baseline and 6-month follow up or 6- and 12-month follow up), (iii) "arising", ie, present at one follow up visit but not at the previous (baseline or 6-month follow-up) visit, and (iv) "no more present", ie, not present at the follow-up visit but present at the previous (baseline or 6-month follow-up) visit.
For descriptive purposes, and for a better contextualization of results, changes of therapies for COPD during study were also evaluated; a therapy was considered changed if interrupted, in case of dosage increase/decrease, or if the patient started a new therapy.

Comparisons between patients included vs not included in the analyses were performed by means of $t$-test or nonparametric Wilcoxon rank sums test for numerical variables and Chi-square test in case of categorical variables. Analysis of variance (for normally distributed variables) and Kruskal-Wallis test by ranks (for non-normally distributed variables) on means and medians, respectively, and Chi-square or Fisher exact test (for categorical variables) were used to compare variables vs m-phenotype (variable in 5 classes). Then, depending on the statistical significance of these tests, Mann-Whitney test on medians (for non-normally distributed variables), $t$-test (for normally distributed variables), and Chi-square or Fisher exact tests (for categorical variables) were applied to compare variables among specific pairs of m-phenotypes. Alpha with Bonferroni correction was set to 0.0001 considering the total number of performed tests.

Correlation between not normally distributed variables was assessed using the Spearman's rank correlation coefficient.

Site monitoring, data management, and statistical analysis were performed by MediNeos (Modena, Italy). Statistical analysis was performed using SAS v9.4 and Enterprise Guide v7.1.

\section{Results \\ Subjects' Characteristics}

Among the 683 COPD-diagnosed patients enrolled in the STORICO study, 77 were excluded because they did not satisfy inclusion/exclusion criteria of the study and another 92 subjects were excluded because of missing information on variables analyzed in the factor and cluster analyses used to identify m-phenotypes; thus, 514 patients were classified at baseline visit into the five m-phenotypes: $\mathrm{MC}, \mathrm{ME}, \mathrm{SB}, \mathrm{SE}$ and SMC.

One-year follow-up data were available for 379 (73.8\%) subjects (144 MC, $71 \mathrm{ME}, 96 \mathrm{SB}, 14 \mathrm{SE}, 54$ SMC) included in the present analysis, whereas 135 patients were not considered evaluable for the analyses, for the reasons detailed in Figure 1.

Patients evaluable for the analyses did not differ at baseline in gender, age, number of COPD exacerbations/ 


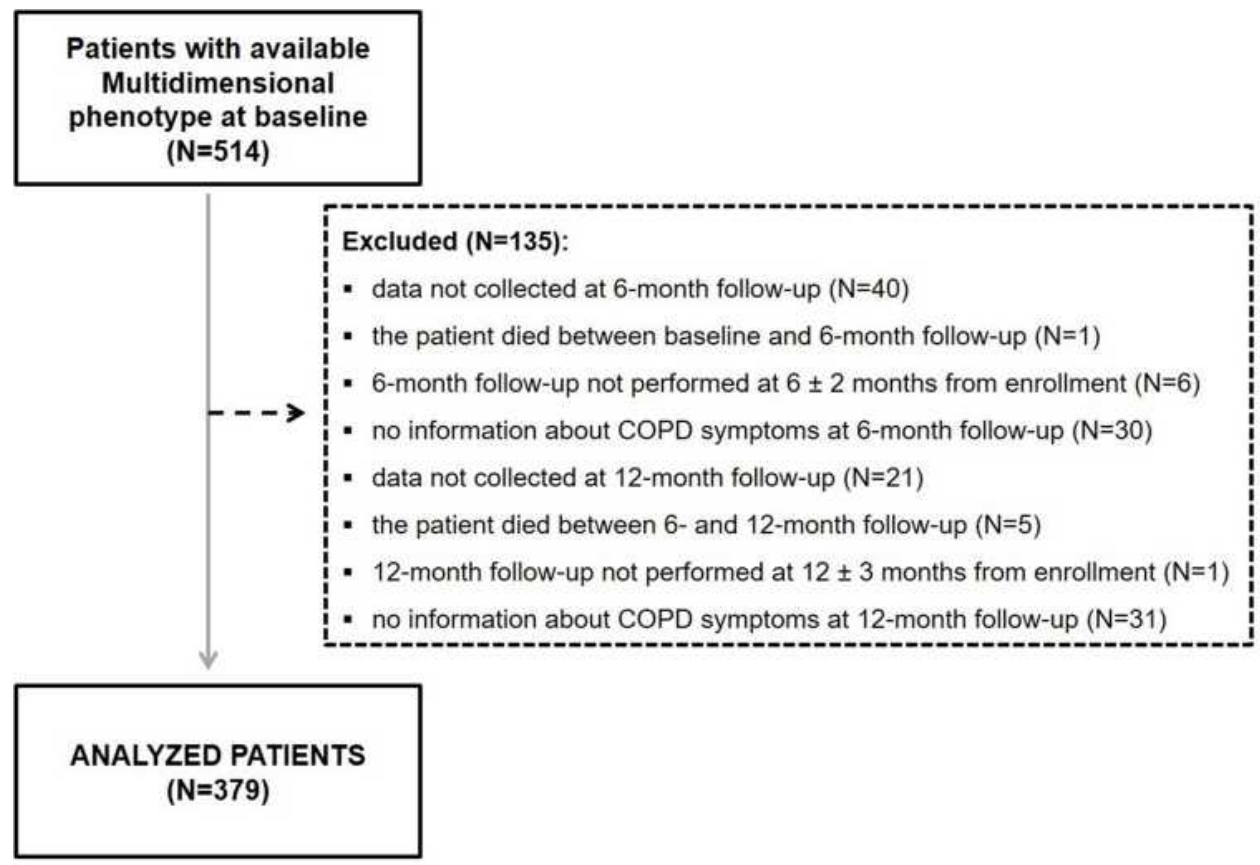

"Data not collected at 6- or 12-month follow up" means that the patient did not attend the study visit. "no information about COPD symptoms at 6- or 12- month follow up" means that the Night-time, Morning and Day-time Symptoms of COPD questionnaire was missing or incomplete.

Figure I Disposition of patients.

year in the 5 years before baseline, FEV1 and DLCO \% predicted, RV, TLC, SGRQ scores compared to not evaluable patients (see Supplementary Table 1). Baseline characteristics of analyzed patients are shown in Table 1 . The distribution of patients according to ongoing therapies for COPD during the study is shown in Table 2. Overall, therapies for COPD were stable during the study with no significant changes between m-phenotypes (Chi-square test p-value presence/absence of changes vs m-phenotype $>0.05)$. Changes of therapies during the study occurred, in fact, in 9 (6.3\%) MC, 5 (7.0\%) ME, 8 (14.8\%) SMC, 11 (11.5\%) SB, and 2 (14.2\%) SE patients.

\section{Circadian Rhythm of Symptoms}

The proportion of patients with at least one night-time, early-morning, and day-time symptom (in the week before visit) at study visits in the different m-phenotypes is reported in Figure 2A-C.

Night-time symptoms were more prevalent in SB, SE, and SMC vs MC and ME m-phenotypes both at baseline (Chi-square test $\mathrm{p}$-value frequency of symptoms at baseline vs m-phenotype $<0.0001$ ) and at follow-up visits (Chi-square test p-value frequency of symptoms at 6-, and 12-month follow-up vs m-phenotype <0.0001), showing a stability of m-phenotypes in their dominant clinical traits.

Symptom prevalence significantly decreased during follow-up in all m-phenotypes (Chi-square test p-values frequency of COPD symptoms at study visits vs m-phenotype <0.0001), in particular in the severe m-phenotypes and more evidently for the nighttime symptoms (Figure 2A-C). During follow-up, 28.1\% $\mathrm{SB}, 50.0 \% \mathrm{SE}$, and $24.1 \% \mathrm{SMC}$ patients with night-time symptoms at baseline had no more symptoms at 6-month follow-up vs $0.7 \% \mathrm{MC}$ and 5.6\% ME patients; $8.3 \% \mathrm{MC}$ and $12.7 \% \mathrm{ME}$ patients without night-time symptoms at baseline reported them at 6-month follow up visit vs $0.0 \%$ in SB, SE, SMC (p-values night-time symptom evolution (baseline $\rightarrow$ 6-month follow-up) $\mathrm{MC}$ vs SB, SE, SMC and ME vs SB, SE, SMC <0.0001) (Table 3).

The proportion of patients with night-time symptoms present at 6-month follow-up and no more symptoms at 12 -month follow-up was $15.6 \%, 14.3 \%, 3.7 \%$ in $\mathrm{SB}, \mathrm{SE}$, and SMC patients, respectively, compared to $4.9 \%$ and $11.3 \%$ in $\mathrm{MC}$ and $\mathrm{ME}$ patients, respectively. Moreover, $10.4 \%, 7.1 \%, 7.4 \% \mathrm{SB}, \mathrm{SE}$, and SMC patients without night-time symptoms at 6-month follow-up had new 
Table I Baseline Characteristics of Analyzed Patients

\begin{tabular}{|c|c|c|c|c|c|}
\hline & $M C(n=144)$ & ME $(n=7 I)$ & SB $(n=96)$ & SE $(n=14)$ & SMC $(n=54)$ \\
\hline Age $(\mathrm{yrs})($ mean $\pm S D)$ & $70.6 \pm 7.3$ & $70.8 \pm 8.1$ & $71.6 \pm 8.1$ & $73.8 \pm 9.6$ & $69.8 \pm+9.1$ \\
\hline Males (N, \%) & $132(77.2)$ & $64(71.9)$ & $91(79.1)$ & $9(60.0)$ & $51(76.1)$ \\
\hline $\begin{array}{l}\text { COPD exacerbations/year } \\
\text { ( } 5 \text { years before baseline) }(\mathrm{N}, \%)\end{array}$ & $n=|7|$ & $n=89$ & $n=115$ & $n=15$ & $n=67$ \\
\hline $0-1$ & $124(72.5)$ & $66(74.2)$ & $70(60.9)$ & $9(60.0)$ & $32(47.8)$ \\
\hline$\geq 2$ & $47(27.5)$ & $23(25.8)$ & $45(39.1)$ & $6(40.0)$ & $35(52.2)$ \\
\hline FEVI of the predicted (\%) (median (IQR)) & $67.0(52.0-83.5)$ & $62.1(54.8-76.0)$ & $63.0(52.5-81.1)$ & $58.5(52.0-71.0)$ & $55.0(41.0-79.0)$ \\
\hline \multirow[t]{2}{*}{ RV (L) (median (IQR)) } & $\mathrm{n}=84$ & $n=45$ & $n=46$ & $n=5$ & $n=28$ \\
\hline & $2.9(2.5-3.7)$ & $3.9(3.4-5.2)$ & $2.9(2.4-3.9)$ & $3.0(2.8-3.1)$ & $2.9(2.3-3.7)$ \\
\hline \multirow[t]{2}{*}{ TLC (L) (mean $\pm S D)$} & $n=82$ & $n=45$ & $n=45$ & $n=5$ & $n=27$ \\
\hline & $6.3 \pm 1.4$ & $7.2 \pm 1.5$ & $6.0 \pm 1.6$ & $6.0 \pm 1.4$ & $5.8 \pm 1.2$ \\
\hline \multirow[t]{2}{*}{ DLCO of the predicted $(\%)($ mean $\pm S D)$} & $n=62$ & $n=32$ & $n=33$ & $n=7$ & $n=15$ \\
\hline & $69.7 \pm 21.2$ & $64.3 \pm 20.9$ & $67.4 \pm 23.5$ & $59.5 \pm 30.5$ & $68.3 \pm 13.6$ \\
\hline SGRQ scores (median (IQR)) & $n=|4|$ & $n=69$ & $n=95$ & $n=12$ & $n=51$ \\
\hline Total & $21.0(14.6-29.7)$ & $32.8(28.0-42.9)$ & $32.6(24.8-45.4)$ & $49.0(34.9-56.0)$ & $53.5(39.5-68.4)$ \\
\hline Symptoms & $24.1(12.4-37.0)$ & $51.1(39.7-60.2)$ & $45.8(32.4-59.0)$ & $53.3(46.5-60.2)$ & $70.9(57.1-78.5)$ \\
\hline Activity & $41.1(23.4-47.7)$ & $48.5(4 \mid .4-66.2)$ & $47.7(35.8-62.2)$ & $66.2(5 I .1-7 \mid .4)$ & $67.1(56.9-85.9)$ \\
\hline Impacts & $10.2(5.0-17.8)$ & $18.7(13.9-28.4)$ & $21.0(11.4-36.3)$ & $35.7(20.1-47.0)$ & $36.9(25.2-61.9)$ \\
\hline
\end{tabular}

Note: If not otherwise specified descriptives were computed over number of patients specified in the column headings.

Abbreviations: DLCO, diffusing capacity of the lung for carbon monoxide; IQR, interquartile range; RV, residual volume; SD, standard deviation; TLC, total lung capacity; MD, mild COPD; ME, mild emphysematous; SB, severe bronchitic; SE, severe emphysematous; SMC, severe mixed COPD.

symptoms at 12 -month follow-up vs $6.3 \%, 2.8 \%$ in $\mathrm{MC}$ and ME (p-values night-time symptom evolution (6-month follow-up $\rightarrow 12$-month follow-up) MC vs SB, SE, SMC and ME vs SB, SE, SMC $<0.0001)$.

The highest prevalence of patients free from earlymorning and day-time symptoms (ie, always absent) was observed in MC compared to other m-phenotypes both at 6 and at 12 months (Table 3).

\section{COPD Exacerbations and Lung Function}

At enrollment, the median (IQR) number of COPD exacerbations/year (in the 5 years before baseline) ranged between $1.0 \quad(0.0-3.0)$ in $\mathrm{MC}$ and 3.0 (1.0-5.0) in SMC patients. The 1-year incidence of exacerbations did not distinguish m-phenotypes: 29 (20.1\%) MC, 22 (31.0\%) ME, 24 (25.0\%) SB, 4 (28.6\%) SE and 18 (33.3\%) SMC had at least one exacerbation (Chi-square test p-value frequency of patients with exacerbations vs m-phenotype $>0.05$ ).

At baseline, the only significant difference in lung function parameters emerged in RV of MC vs ME patients (median (IQR): $2.9 \quad(2.5-3.7)$ L vs 3.9 (3.4-5.2) L; Mann-Whitney test p-value (MC vs ME) $<0.0001$ ) (see Supplementary Table 2). Between baseline and 6-month follow-up, TLC decreased more in ME patients (median (IQR): $-1.4(-2.3-0.2) \mathrm{L}$ ) than in SB (median (IQR): $0.1(-0.2-0.8)$ L; Mann-Whitney test $\mathrm{p}$-value (ME vs $\mathrm{SB})<0.0001$ ). The frequency of patients with dyspnea at different time points is reported in Supplementary Table 2; it appears to be stable during time within m-phenotypes. The level of dyspnea is significantly lower at study visits in MC compared to other m-phenotypes (Chi-square test p-value presence of dyspnea in MC vs other m-phenotypes $<0.0001)$. 
Table 2 Therapies for COPD Ongoing at Study Visits (by Multidimensional Phenotype)

\begin{tabular}{|c|c|c|c|c|c|}
\hline & $M C(n=144)$ & ME $(n=71)$ & SB $(n=96)$ & SE $(n=14)$ & SMC $(n=54)$ \\
\hline \multicolumn{6}{|l|}{ Baseline (n, \%) } \\
\hline Triple therapy & $40(27.8)$ & $27(38.0)$ & $44(45.8)$ & $3(2 \mid .4)$ & $19(35.2)$ \\
\hline LABA+LAMA & $39(27.1)$ & $22(31.0)$ & $20(20.8)$ & $4(28.6)$ & $18(33.3)$ \\
\hline LAMA Alone & $35(24.3)$ & II (I5.5) & $12(12.5)$ & I (7.I) & $8(14.8)$ \\
\hline ICS+LABA & $22(15.3)$ & $6(8.5)$ & $16(16.7)$ & $5(35.7)$ & $7(13.0)$ \\
\hline Other & $8(5.5)$ & $5(7.0)$ & $4(4.1)$ & I (7.I) & $2(3.7)$ \\
\hline \multicolumn{6}{|c|}{ 6-month follow-up (n, \%) } \\
\hline Triple therapy & $39(27.1)$ & $27(38.0)$ & $45(46.9)$ & $4(28.6)$ & $19(35.2)$ \\
\hline LABA+LAMA & $42(29.2)$ & $23(32.4)$ & $21(21.9)$ & $5(35.7)$ & $19(35.2)$ \\
\hline LAMA Alone & $34(236)$ & $12(16.9)$ & $10(10.4)$ & I (7.I) & $7(13.0)$ \\
\hline$I C S+L A B A$ & $21(14.6)$ & $5(7.0)$ & $16(16.7)$ & $3(21.4)$ & $8(14.8)$ \\
\hline Other & $8(5.5)$ & $4(5.6)$ & $4(4.1)$ & I (7.I) & I (I.9) \\
\hline \multicolumn{6}{|c|}{ I 2-month follow-up (n, \%) } \\
\hline No ongoing therapies & $\mathrm{I}(0.7)$ & $0(0.0)$ & $0(0.0)$ & $0(0.0)$ & $0(0.0)$ \\
\hline Triple therapy & $38(26.4)$ & $26(36.6)$ & $46(47.9)$ & $4(28.6)$ & $19(35.2)$ \\
\hline LABA+LAMA & $43(29.9)$ & $23(32.4)$ & $20(20.8)$ & $5(35.7)$ & $19(35.2)$ \\
\hline LAMA Alone & $33(22.9)$ & $12(16.9)$ & II (II.5) & I (7.I) & $7(13.0)$ \\
\hline ICS+LABA & $21(14.6)$ & $6(8.5)$ & $16(16.7)$ & $3(2 \mid .4)$ & $8(14.8)$ \\
\hline Other & $8(5.5)$ & $4(5.6)$ & $3(3.1)$ & I (7.I) & I (I.9) \\
\hline
\end{tabular}

Notes: Percentages were computed over number of patients specified in the column headings. Triple therapy is any combination of ICS, LAMA and LABA in fixed dose combination or not; Other: SABA, LABA Alone, ICS+LABA, ICS+SABA, ICS+LAMA.

Abbreviations: ICS, inhaled corticosteroids; LABA, long-acting $\beta 2$-agonists; LAMA, long-acting muscarinic agonists; SAMA, short-acting muscarinic antagonist; SABA, short-acting $\beta 2$-agonists; MD, mild COPD; ME, mild emphysematous; SB, severe bronchitic; SE, severe emphysematous2; SMC, severe mixed COPD.

\section{Quality of Life and Sleep, Anxiety/ Depression}

The quality of life did not change during 12 months of observation, irrespective of m-phenotype. It was always better in MC (Mann-Whitney test p-values (MC vs ME, $\mathrm{SB}, \mathrm{SE}, \mathrm{SMC})<0.0001$ for SGRQ symptoms, activity, impacts and total scores at baseline, 6-month and 12month follow-up). Quality of life was worse in SMC (Mann-Whitney test p-value (SMC vs ME and SB) $<0.0001$ for symptoms, activity, impacts and total scores at baseline, 6-month and 12-month follow-up) (see Supplementary Table 3).

At all visits, MC had better quality of sleep than the other m-phenotypes (Mann-Whitney test p-values CASIS total score in MC vs other m-phenotypes $<0.0001$ ), while
SMC had a worse quality of sleep compared to ME and SB patients (Mann-Whitney test p-value CASIS total score SMC vs ME and SB <0.0001) (see Supplementary Table 3). Between baseline and follow-up visits, all m-phenotypes showed improved quality of sleep, more markedly in severe (SB, SE, SMC) compared to mild (MC, ME) phenotypes (Mann-Whitney test p-values change of CASIS total score between baseline and 6- or 12-month follow-up in MC, ME vs SB, SE, SMC $<0.0001)$.

Perceived quality of life did not improve with improving quality of sleep (Spearman correlation coefficients of change baseline-6-month follow-up and baseline-12month follow-up of CASIS total score vs SGRS activity, impacts, symptoms, total scores $<0.3$ ). 


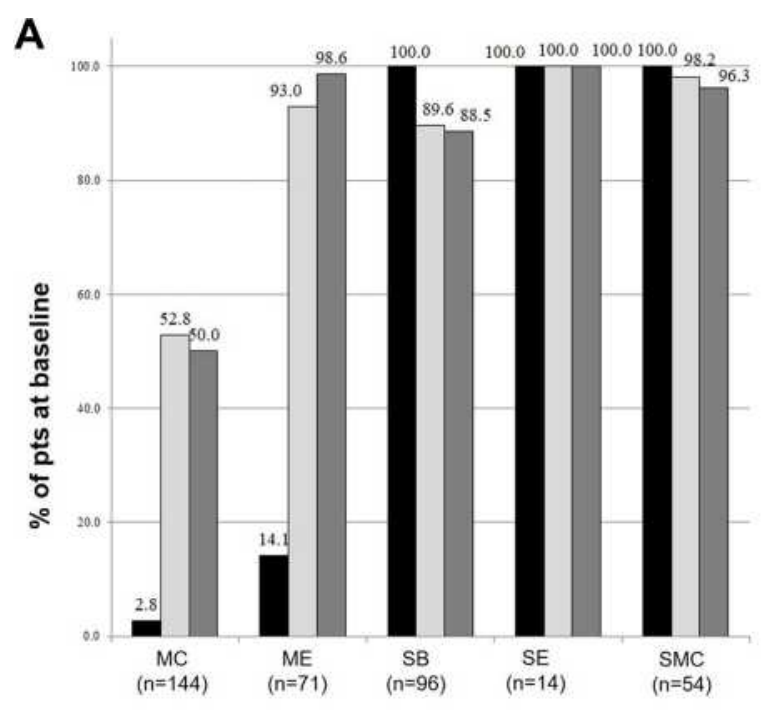

Night-time
Early-morning Day-time

\section{B}

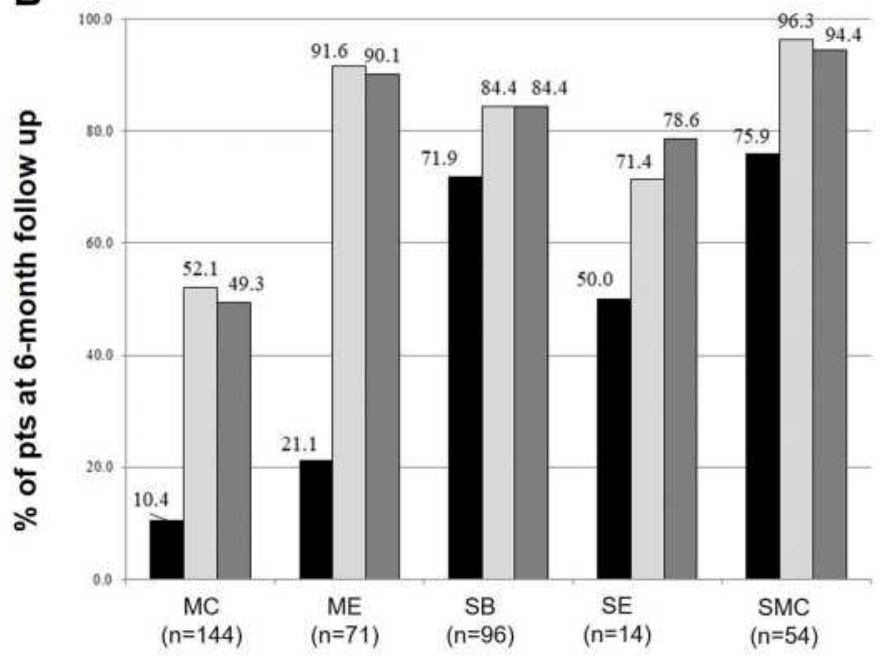

Night-time

Early-morning
Day-time

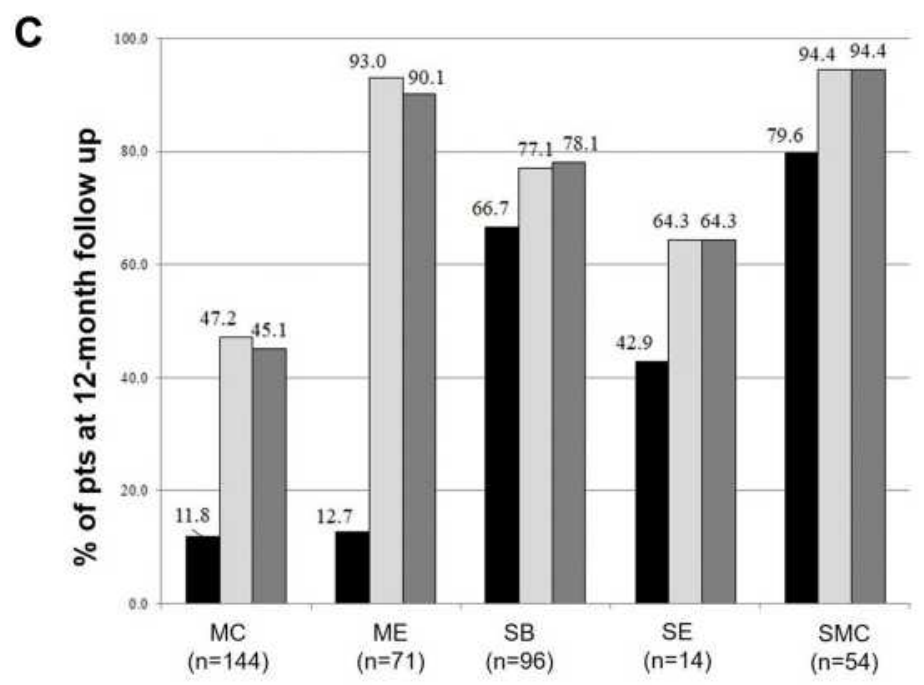

Night-time $\square$ Early-morning $\square$ Day-time

Figure 2 (A) Frequency of patients with COPD symptoms by multidimensional phenotype at baseline. (B) Frequency of patients with COPD symptoms by multidimensional phenotype at 6-month follow-up. (C) Frequency of patients with COPD symptoms by multidimensional phenotype at I2-month follow-up.

At all study visits, levels of anxiety and depression were low (see Supplementary Table 3). SMC had higher levels of anxiety and depression than $\mathrm{MC}$ and SB (Mann-Whitney test p-values HADS scores at all study visits in $\mathrm{SMC}$ vs $\mathrm{MC}, \mathrm{SB}<0.0001)$. Significant differences between $\mathrm{ME}$ and $\mathrm{MC}$ also emerged during the study in favor of MC (Mann-Whitney test p-values HADS scores at all study visits in $\mathrm{ME}$ vs $\mathrm{MC}$ $<0.0001)$. HADS scores appeared stable over time in all m-phenotypes.

\section{Discussion}

We found that m-phenotypes are characterized by stable distinctive clinical (ie, presence of symptoms) and health status (ie, quality of life, quality of sleep, level of anxiety and depression) features. Night-time symptoms were more prevalent in SB, SE and SMC vs MC and ME m-phenotypes both at baseline and at follow-up visits and SMC constantly had higher levels of anxiety and depression than $\mathrm{MC}$ and SB. Thus, contrasting anxiety and depression seems an objective worthy of 
Table 3 I-Year Evolution of COPD Symptoms (by Multidimensional Phenotype)

\begin{tabular}{|c|c|c|c|c|c|c|}
\hline & $M C(n=\mid 44)$ & ME $(n=7 I)$ & SB $(n=96)$ & SE $(n=14)$ & SMC $(n=54)$ & p-value \\
\hline \multicolumn{7}{|c|}{ Night-time symptoms evolution ( $\mathrm{n}, \%)$} \\
\hline \multicolumn{7}{|c|}{ Between baseline and 6-month follow-up } \\
\hline Arising & $12(8.3)$ & $9(12.7)$ & $0(0.0)$ & $0(0.0)$ & $0(0.0)$ & $<0.0001$ \\
\hline Always absent & $128(88.9)$ & $52(73.2)$ & $0(0.0)$ & $0(0.0)$ & $0(0.0)$ & \\
\hline Always present & $3(2.1)$ & $6(8.5)$ & $69(71.9)$ & $7(50.0)$ & $41(75.9)$ & \\
\hline No more present & $\mathrm{I}(0.7)$ & $4(5.6)$ & $27(28.1)$ & $7(50.0)$ & $13(24.1)$ & \\
\hline \multicolumn{7}{|c|}{ Between 6-month and I2-month follow-up } \\
\hline Arising & $9(6.3)$ & $2(2.8)$ & $10(10.4)$ & I (7.I) & $4(7.4)$ & $<0.0001$ \\
\hline Always absent & $120(83.3)$ & $54(76.1)$ & $17(17.7)$ & $6(42.9)$ & $9(16.7)$ & \\
\hline Always present & $8(5.6)$ & $7(9.9)$ & $54(56.3)$ & $5(35.7)$ & $39(72.2)$ & \\
\hline No more present & $7(4.9)$ & $8(11.3)$ & $15(15.6)$ & $2(14.3)$ & $2(3.7)$ & \\
\hline \multicolumn{7}{|c|}{ EARLY-MORNING symptoms evolution (n, \%) } \\
\hline \multicolumn{7}{|c|}{ Between baseline and 6-month follow-up } \\
\hline Arising & II (7.6) & $5(7.0)$ & $2(2.1)$ & $0(0.0)$ & I (I.9) & $<0.0001$ \\
\hline Always absent & $57(39.6)$ & $0(0.0)$ & $8(8.3)$ & $0(0.0)$ & $0(0.0)$ & \\
\hline Always present & $64(44.4)$ & $60(84.5)$ & $79(82.3)$ & $10(7 \mid .4)$ & $51(94.4)$ & \\
\hline No more present & $12(8.3)$ & $6(8.5)$ & $7(7.3)$ & $4(28.6)$ & $2(3.7)$ & \\
\hline \multicolumn{7}{|c|}{ Between 6-month and I2-month follow-up } \\
\hline Arising & II (7.6) & $4(5.6)$ & $5(5.2)$ & $2(14.3)$ & $2(3.7)$ & $<0.0001$ \\
\hline Always absent & $58(40.3)$ & $2(2.8)$ & $10(10.4)$ & $2(14.3)$ & $0(0.0)$ & \\
\hline Always present & $57(39.6)$ & $62(87.3)$ & $69(71.9)$ & $7(50.0)$ & $49(90.7)$ & \\
\hline No more present & $18(12.5)$ & $3(4.2)$ & $12(12.5)$ & $3(2 I .4)$ & $3(5.6)$ & \\
\hline \multicolumn{7}{|c|}{ DAY-TIME symptoms evolution ( $\mathrm{n}, \%)$} \\
\hline \multicolumn{7}{|c|}{ Between baseline and 6-month follow-up } \\
\hline Arising & $12(8.3)$ & I ( $(1.4)$ & $5(5.2)$ & $0(0.0)$ & I (I.9) & $<0.0001$ \\
\hline Always absent & $60(41.7)$ & $0(0.0)$ & $6(6.3)$ & $0(0.0)$ & I (I.9) & \\
\hline Always present & $59(4 I .0)$ & $63(88.7)$ & $76(79.2)$ & II (78.6) & $50(92.6)$ & \\
\hline No more present & $13(9.0)$ & $7(9.9)$ & $9(9.4)$ & $3(21.4)$ & $2(3.7)$ & \\
\hline \multicolumn{7}{|c|}{ Between 6-month and I 2-month follow-up } \\
\hline Arising & $12(8.3)$ & $3(4.2)$ & $5(5.2)$ & $0(0.0)$ & $3(5.6)$ & $<0.0001$ \\
\hline Always absent & $61(42.4)$ & $4(5.6)$ & $10(10.4)$ & $3(2 \mid .4)$ & $0(0.0)$ & \\
\hline Always present & $53(36.8)$ & $61(85.9)$ & $70(72.9)$ & $9(64.3)$ & $48(88.9)$ & \\
\hline No more present & $18(12.5)$ & $3(4.2)$ & II (II.5) & $2(14.3)$ & $3(5.6)$ & \\
\hline
\end{tabular}

Note: Percentages were computed over number of patients specified in the column headings.

Abbreviations: MD, mild COPD; ME, mild emphysematous; SB, severe bronchitic; SE, severe emphysematous; SMC, severe mixed COPD. 
consideration in SMC m-phenotype. Analogously, showing that SMC constantly had the worst perceived health status might drive dedicated interventions. Indeed, classifying COPD patients according to the m-phenotype would enable physicians to measure the effects of a given intervention on the main m-phenotype specific problems of the individual patient.

The current longitudinal analysis also showed that severe m-phenotypes had greater improvement in nocturnal symptoms as opposed to mild m-phenotypes. Moreover, quality of sleep improved across all m-phenotypes, more markedly in severe than mild ones. On the other hand, the prevalence of early-morning and day-time symptoms remained high, with the majority of study participants $(78.1 \%$ of SB, $64.3 \%$ of SE, and $94.4 \%$ of SMC) still suffering from day-time symptoms one year after enrolment. The lack of improvement in dyspnea and quality of life testifies to this unfavorable trend. Thus, the improvements in nocturnal symptoms and quality of sleep did not translate into better healthrelated quality of life.

Once a forgotten dimension of COPD, nocturnal symptoms have become a specific target of pharmacological treatment. In a real-world treatment setting, ${ }^{24}$ sleep disturbances were common and, if left unmanaged, encroached on daytime activities, worsening health status. Indeed, night-time symptoms have been proved to be the main correlate of depressed mood in the Assess study. ${ }^{18}$ Furthermore, in a mixed Asthma-COPD population aged over 65 years, their prevalence increases with age. ${ }^{25}$ Of note, in the CanCOLD study ${ }^{26}$ impaired sleep quality was associated with increased rate of exacerbations over a period of 18 months. Finally, sleep disturbances have recently been proved to correlate with respiratory symptoms, poor lung function, exacerbations during the previous year, and depression in a large COPD cohort.

We assessed the evolution of respiratory symptoms in COPD patients in real-life, ie, in the absence of planned interventions. This condition may be, to some extent, compared to the placebo arm of RCTs, with regard to indexes exploring health status, dyspnea, and respiratory function tests; however, the evolution of the circadian rhythm of symptoms has never been assessed so far. In the placebo group of the two largest trials on the pharmacological treatment of COPD, namely the UPLIFT and TORCH studies, neither health status nor lung function showed clinically significant changes over a 4- and 3-year interval, respectively. ${ }^{26-28}$ Similar findings were documented for perceived dyspnea in the pooled Flight 1 and Flight 2 trials after one year of observation. ${ }^{29}$ Finally, in a real-world population of COPD patients in conditions of usual care (the SALFORD study), overall dyspnea did not change significantly as it improved in $26.8 \%$ of patients but worsened in $20.9 \%$ over 12 months. ${ }^{30}$ Thus, our findings about the evolution of respiratory symptoms are in line with comparable observations centered on overall symptoms and not on their circadian rhythm.

Surprisingly, in our patients' quality of life did not improve with improving quality of sleep. This likely reflects the dominating role of activities in the SGRQ; indeed, this scale largely relies upon the physical dimension -ie, basic and instrumental activities of daily livingwhich mainly depends upon diurnal rather than nocturnal symptoms, and no question explores quality of sleep or sleep-related symptoms. This very likely explains our finding, ie, the decoupling between nocturnal symptoms/quality of sleep and health-related quality of life and this decoupling cautions against overestimating the potential effects of improving nocturnal control of airway obstruction.

Alternatively, one might hypothesize that in ours and in previous series, dyspnea -which is strictly related to activity-, is the main determinant of perceived health status. Finally, it cannot be excluded that m-phenotypes also reflect some constitutive affective trait conditioning enteroception and mood.

The reasons accounting for the positive evolution of nocturnal symptoms in a notable fraction of patients, mainly belonging to the severe m-phenotypes, are unclear. Indeed, pharmacological therapy changed marginally during follow-up with no relevant differences between m-phenotypes. Average change of SGRQ scores between study visits was negligible due to diverging and reciprocally compensating changes within a class. Some "research effect", ie, a greater attention to optimize the care of enrollees, cannot be excluded. Furthermore, we lacked information on non-pharmacological therapies such as rehabilitation, which might have contributed to modify the symptoms. Nevertheless, day-time symptoms showed a less positive trend as if effort-related symptoms were less modifiable than sleep-related ones. Indeed, nocturnal symptoms variously reflect changes in bronchial tone, defective clearance of mucus and late onset symptoms, waning pharmacological effects, all of which are modifiable through a tailored approach in terms of timing and choice of therapy. Day-time symptoms, instead, are 
primarily related to effort-related ventilatory demand. Fulfilling such a demand requires efficient gas exchanges and bronchial patency, which may partly benefit from improved pharmacological therapy, but also preserved muscle mass and strength, and adaptative mechanisms of the breathing pattern to the physical effort. These latter determinants of the response to exercise are less likely to improve in response to pharmacological measures. Furthermore, selected comorbidities like anemia or renal failure might contribute to blunt the response to exercise. These mechanisms might underlie the decoupling in the evolutions of nocturnal and day-time symptoms in our patients. Finally, this decoupling seems to exclude that improvement of nocturnal symptoms in the most diseased patients reflects regression to the mean.

Exacerbations were distinctly rare in our populations. Interestingly, the rate of exacerbations in a populationbased sample of a longitudinal observational investigation (CanCOLD study) was 0.50 per year, ${ }^{26}$ in the entire trial population of the SALFORD study, which had selecting criteria comparable to those of the STORICO study, 31\% of patients had no COPD exacerbations in the year of follow-up. ${ }^{31}$ These findings likely reflect a secular trend: in the recent TRIBUTE study, patients with severe to very severe COPD and a reported exacerbation rate of 1.2 per year actually had 0.5 and 0.59 exacerbations over one year while assuming triple or dual bronchodilating therapy. ${ }^{32}$ Analogously, the rate of exacerbations dramatically declined in patients enrolled and treated with triple or double Trilogy. ${ }^{33}$ In a large survey of over 99574 COPD patients up to 10 years, the vast majority had no exacerbations in the baseline year and a quarter of the patients remained free from exacerbations over the whole 10-year observation period. ${ }^{34}$ An improvement of the whole therapeutic approach to COPD is likely to account for this favorable trend.

Our work has some limitations. First, a large fraction of enrolled patients was excluded from the analyses due to missing information. However, followed-up and excluded patients did not differ in baseline characteristics. Second, we collected symptoms throughout the 24 hours, but we had no measure of symptom severity available. Theoretically, an unchanged prevalence of symptoms in a given time frame might correspond to a different intensity of symptoms. However, the fact that both dyspnea (mMRC) and quality of life (SGRQ) did not change over time is consistent with the recorded symptoms being stable in intensity over time. Third, the improvement of night-time symptoms in a substantial fraction of severe m-phenotype patients might, at least to some extent, depend upon the effective treatment of non-respiratory causes of disturbed sleep like urologic and affective problems. Indeed, we could not assess this possibility. Finally, present data are worthy of being confirmed in a testing population. Thus, they should be considered preliminary in nature. However, based on the real life and purely observational dimension of this study, they are very likely to reflect a biologically plausible and clinically convenient clustering of COPD patients.

\section{Conclusions}

Over a 1-year period, a considerable decrease of nighttime symptoms occurred in severe m-phenotypes confirmed by the evolution of CASIS score, but sleep improvement did not translate in better quality of life and day-time symptoms continued to be highly prevalent at the end of the follow-up.

The m-phenotypes define clusters of patients with stable and distinctive health status profiles and clinical trajectories and, thus, might help tailor therapeutic interventions and measure therapy effects with regard to the specific characteristics of the m-phenotype. Furthermore, according to these preliminary data, the proposed m-phenotype, a symptom- and QoL-based classification of COPD patients, seems worthy of testing also for research purposes. Indeed, designing a multi-outcome trial, ie, one measuring different outcomes for different m-phenotypes, might improve trial design and results by capturing what is of primary interest for the individual patient. Thus, m-phenotypes might become a working tool in the future of precision medicine.

\section{Abbreviations}

CASIS, COPD and Asthma Sleep Impact Scale; COPD, chronic obstructive pulmonary disease; DLCO, diffusion capacity for carbon monoxide; FEV1, forced expiratory volume in the first second; HADS, Hospital Anxiety and Depression Scale; ICS, inhaled corticosteroids; IQR, interquartile range; LABA, long-acting $\beta 2$-agonists; LAMA, long-acting muscarinic agonists; $\mathrm{MC}$, mild COPD; ME, mild emphysematous; mMRC, modified Medical Research Council; RV, residual volume; SB, severe bronchitic; SD, standard deviation; SE, severe emphysematous; SGRQ, St. George's Respiratory Questionnaire; SMC, severe mixed COPD; TLC, total lung capacity. 


\section{Data Sharing Statement}

The data supporting the findings of this study are available from Raffaele Antonelli Incalzi, Francesco Blasi, Nicola Scichilone, Alessandro Zullo, Lucia Simoni, Giorgio Walter Canonica and Laboratori Guidotti, Italy but restrictions apply to the availability of these data, which were used under license for the current study, and are therefore not publicly available. There are legal and ethical restrictions since data contain potentially sensitive patient information. Data are however available from ì Raffaele Antonelli Incalzi, Francesco Blasi, Nicola Scichilone, Alessandro Zullo, Lucia Simoni, Giorgio Walter Canonica and Laboratori Guidotti upon reasonable request. Data request may be sent to the first author (r.antonelli@unicampus.it) and to Stefania Barsanti (Laboratori Guidotti, sbarsanti@labguidotti.it).

\section{Acknowledgments}

We are grateful to Sara Rizzoli for her help in analyzing data and drafting the manuscript. We are also grateful to the participating centers, involved in data collection.

Members of the STORICO Study Group Participating centers.

Pietro Schino (Acquaviva delle Fonti); Giuseppina Cuttitta (Palermo); Maria Pia Foschino (Foggia); Renato Prediletto (Pisa); Carmelindo Mario Enrico Tranfa (Napoli); Maria Cristina Zappa (Roma); Pasquale Patriciello (Pollena Trocchia); Luciana Labate (Bari); Salvatore Mariotta (Roma); Stefano Nava (Bologna); Alessandro Vatrella (Salerno); Michele Mastroberardino (Avellino); Riccardo Sarzani (Ancona); Antonio Iuliano (Milano); Lamberto Maggi (Bergamo); Anna Zedda (Casoria); Alberto Pesci (Monza); Giuseppe Sera (Torino); Antonello Nicolini (Sestri Levante); Salvatore Walter Di Donato (Mondragone); Silvia Forte (Roma); Mario Del Donno (Benevento); Federica Rivolta (Abbiategrasso); Mauro Ferliga (Chiari); Antonio Filippo Raco (Menaggio); Luigi Di Re (Teramo); Gaetano Cabibbo (Modica); Rosario Maselli (Catanzaro); Carlo Gulotta (Orbassano); Stefano Nardini (Vittorio Veneto); Enrico Eugenio Guffanti (Casatenovo); Walter Castellani (Firenze); Luca Triolo (Roma); Giovanni Passalacqua (Messina); Bianca Beghè (Modena); Salvatore Lo Cicero (Milano); Enzo Faccini (Dolo); Elena Atzeni (Nuoro); Roberto Tazza (Terni); Piercarlo Giamesio (Asti).

\section{Author Contributions}

All authors made a significant contribution to the work reported, either in the conception, study design, execution, acquisition of data, analysis and interpretation, or in all these areas; they have been involved in drafting, revising or critically reviewing the article; they have approved the final version to be published, have chosen the journal to which the article has been submitted and have agreed to be accountable for all aspects of the work.

\section{Funding}

The study was supported by a grant from Laboratori Guidotti and Malesci, Italy. The funding sources were not involved in study design, in the collection, analysis and interpretation of data, and in the preparation of the article for publication.

\section{Disclosure}

Francesco Blasi reports grants and personal fees from AstraZeneca, grants and personal fees from BAYER, grants and personal fees from CHIESI, personal fees from GSK, personal fees from GUIDOTTI, grants and personal fees from GRIFOLS, grants from INSMED, personal fees from MENARINI, personal fees from NOVARTIS, grants and personal fees from PFIZER, personal fees from TEVA, grants from VERTEX, personal fees from ZAMBON, outside the submitted work. Alessandro Zullo and Lucia Simoni are employees of Medineos Observational Research. The other authors declare that they have no competing interests.

\section{References}

1. Lange P, Halpin DM, O'Donnell DE, MacNee W. Diagnosis, assessment, and phenotyping of COPD: beyond FEV1. Int J Chron Obstruct Pulmon Dis. 2016;11 Spec Iss:3-12. doi:10.2147/COPD.S85976

2. Wan ES, Castaldi P, Cho MH, et al. B13 any time at all: disease progression and emerging phenotypes in COPD. Unsupervised clustering methods predict future development COPD among subjects with preserved ratio impaired spirometry (PRISm) in the COPDGene study. Am J Respir Crit Care Med. 2015;191:1.

3. Burgel PR, Paillasseur JL, Caillaud D, et al. Clinical COPD phenotypes: a novel approach using principal component and cluster analyses. Eur Respir J. 2010;36(3):531-539. doi:10.1183/ 09031936.00175109

4. Burgel PR, Paillasseur JL, Peene B, et al. Two distinct chronic obstructive pulmonary disease (COPD) phenotypes are associated with high risk of mortality. PLoS One. 2012;7(12):e51048. doi:10.1371/journal.pone.0051048

5. Celli BR, Locantore N, Tal-Singer R, et al. Emphysema and extrapulmonary tissue loss in COPD: a multi-organ loss of tissue phenotype. Eur Respir J. 2018;51(2):pii: 1702146. doi:10.1183/ 13993003.02146-2017 
6. Agusti A. The disease model: implications for clinical practice. Eur Respir J. 2018;51(4):pii: 1800188. doi:10.1183/13993003.001882018

7. Miravitlles M, Calle M, Soler-Cataluna JJ. Clinical phenotypes of COPD: identification, definition and implications for guidelines. Arch Bronconeumol. 2012;48(3):86-98. doi:10.1016/j.arbr.2012.01.003

8. Calle Rubio M, Casamor R, Miravitlles M. Identification and distribution of COPD phenotypes in clinical practice according to Spanish COPD guidelines: the FENEPOC study. Int $J$ Chron Obstruct Pulmon Dis. 2017;12:2373-2383. doi:10.2147/COPD. S137872

9. Agusti A, Bel E, Thomas M, et al. Treatable traits: toward precision medicine of chronic airway diseases. Eur Respir J. 2016;47 (2):410-419. doi:10.1183/13993003.01359-2015

10. Garcia Aymerich J, Gómez FP, Benet M, et al. Identification and prospective validation of clinically relevant chronic obstructive pulmonary disease (COPD) subtypes. Thorax. 2011;66(5):430-437. doi:10.1136/thx.2010.154484

11. Burgel PR, Paillasseur JL, Roche N. Identification of clinical phenotypes using cluster analyses in COPD patients with multiple comorbidities. Biomed Res Int. 2014;2014:420134. doi:10.1155/ 2014/420134

12. Esteban C, Arostegui I, Aburto M, et al. Chronic obstructive pulmonary disease subtypes. Transitions over time. PLoS One. 2016;11 (9):e0161710. doi:10.1371/journal.pone.0161710

13. Augustin IML, Spruit MA, Houben-Wilke S, et al. The respiratory physiome: clustering based on a comprehensive lung function assessment in patients with COPD. PLoS One. 2018;13(9):e0201593. doi:10.1371/journal.pone.0201593

14. Yohannes AM, Alexopoulos GS. Depression and anxiety in patients with COPD. Eur Respir Rev. 2014;23(133):345-349. doi:10.1183/ 09059180.00007813

15. Canonica GW, Blasi F, Scichilone N, et al. Characterization of circadian COPD symptoms by phenotype: methodology of the STORICO observational study. Eur J Intern Med. 2017;43:62-68. doi:10.1016/j.ejim.2017.05.021

16. Agusti A, Hedner J, Marin JM, Barbé F, Cazzola M, Rennard S. Night-time symptoms: a forgotten dimension of COPD. Eur Respir Rev. 2011;20(121):183-194. doi:10.1183/09059180.00004311

17. Antonelli Incalzi R, Canonica GW, Scichilone N, et al. The COPD multi-dimensional phenotype: a new classification from the STORICO Italian observational study. PLoS One. 2019;14(9): e0221889. doi:10.1371/journal.pone.0221889

18. Miravitlles M, Worth H, Soler Catalauña JJ, et al. Observational study to characterize 24-hour COPD symptoms and their relationship with patient-reported outcomes; results from the ASSESS study. Respir Res. 2014;21(15):122. doi:10.1186/s12931-0140122-1

19. Bestall JC, Paul EA, Garrod R, et al. Usefulness of the Medical Research Council (MRC) dyspnoea scale as a measure of disability in patients with chronic obstructive pulmonary disease. Thorax. 1999;54(7):581-586. doi:10.1136/thx.54.7.581

20. Jones PW, Quirk FH, Baveystock CM, Littlejohns P. A self-complete measure for chronic airflow limitation. The St George's respiratory questionnaire. Am Rev Respir Dis. 1992;145(6):1321-1327. doi:10.1164/ajrccm/145.6.1321
21. Meguro M, Barley EA, Spencer S, Jones PW. Development and validation of an improved COPD-specific version of the $\mathrm{St}$ George's respiratory questionnaire. Chest. 2007;132(2):456-463. doi:10.1378/chest.06-0702

22. Zigmond AS, Snaith RP. The hospital anxiety and depression scale. Acta Psychiatr Scand. 1983;67(6):361-370. doi:10.1111/j.16000447.1983.tb09716.x

23. Pokrzywinski RF, Meads DM, McKenna SP, Glendenning GA, Revicki DA. Development and psychometric assessment of the COPD and Asthma Sleep Impact Scale (CASIS). Health Qual Life Outcomes. 2009;7(1):98. doi:10.1186/1477-7525-7-98

24. Ding B. Smal M Treatment trends in patients with asthma-COPD overlap syndrome in a COPD cohort: findings from a real-world survey. Int J Chron Obstruct Pulmon Dis. 2017;12:1753-1763. doi:10.2147/COPD.S136314

25. Bellia V, Catalano F, Scichilone N, et al. Sleep disorders in the elderly with and without chronic airflow obstruction: the SARA study. Sleep. 2003;26(3):318-323. doi:10.1093/sleep/26.3.318

26. Shorofsky M, Bourbeau J, Kimoff J, et al. Impaired sleep quality in COPD is associated with exacerbations: the CanCOLD cohort study. Chest. 2019;156(5):852-863.

27. Tashkin DP, Celli B, Senn S, et al. A 4-year trial of tiotropium in chronic obstructive pulmonary disease. $N$ Engl J Med. 2008;359 (15):1543-1554. doi:10.1056/NEJMoa0805800

28. Calverley PM, Anderson JA, Celli B, et al. Salmeterol and fluticasone propionate and survival in chronic obstructive pulmonary disease. $N$ Engl J Med. 2007;356(8):775-789. doi:10.1056/NEJMoa063070

29. Mahler DA, Kerwin E, Ayers T, et al. FLIGHT1 and FLIGHT2: efficacy and safety of QVA149 (Indacaterol/Glycopyrrolate) versus its monocomponents and placebo in patients with chronic obstructive pulmonary disease. Am J Respir Crit Care Med. 2015;192 (9):1068-1079. doi:10.1164/rccm.201505-1048OC

30. Doward L, Svedsater H, Whalley D, et al. Salford Lung Study in chronic obstructive pulmonary disease (SLS COPD): follow-up interviews on patient-centred outcomes. NPJ Prim Care Resp Med. 2017;22:66. doi:10.1038/s41533-017-0066-2

31. Whalley D, Svedsater H, Doward L, et al. Follow-up interviews from The Salford Lung Study (COPD) and analyses per treatment and exacerbations. NPJ Prim Care Respir Med. 2019;29(1):20. doi:10.1038/s41533-019-0123-0

32. Papi A, Vestbo J, Fabbri L, et al. Extrafine inhaled triple therapy versus dual bronchodilator therapy in chronic obstructive pulmonary disease (TRIBUTE): a double-blind, parallel group, randomised controlled trial. The Lancet. 2018;391(10125):1076-1084. doi:10.1016/ S0140-6736(18)30206-X

33. Singh D, Papi A, Corradi M, et al. Single inhaler triple therapy versus inhaled corticosteroid plus long-acting $\beta 2$-agonist therapy for chronic obstructive pulmonary disease (TRILOGY): a double-blind, parallel group, randomised controlled trial. The Lancet. 2016;388 (10048):963-973. doi:10.1016/S0140-6736(16)31354-X

34. Rothnie KJ, Müllerová H, Smeeth L, Quint JK. Natural history of chronic obstructive pulmonary disease exacerbations in a general practice-based population with chronic obstructive pulmonary disease. Am J Respir Crit Care Med. 2018;198(4):464-471. doi:10.1164/ rccm.201710-2029OC 


\section{Publish your work in this journal}

The International Journal of COPD is an international, peer-reviewed journal of therapeutics and pharmacology focusing on concise rapid reporting of clinical studies and reviews in COPD. Special focus is given to the pathophysiological processes underlying the disease, intervention programs, patient focused education, and self management protocols. This journal is indexed on PubMed Central, MedLine and CAS. The manuscript management system is completely online and includes a very quick and fair peer-review system, which is all easy to use. Visit http://www.dovepress.com/testimonials.php to read real quotes from published authors. 